\title{
RANDOM DYNAMICAL SYSTEMS, ENTROPIES AND INFORMATION
}

\author{
Maurizio Serva \\ Istituto Nazionale di Fisica della Materia and Dipartimento di Matematica \\ Università dell'Aquila, I-67010 Coppito, L'Aquila, Italy
}

(July 3, 2018)

\begin{abstract}
Prediction of events is the challenge in many different disciplines, from meteorology to finance; the more this task is difficult, the more a system is complex. Nevertheless, even according to this restricted definition, a general consensus on what should be the correct indicator for complexity is still not reached. In particular, this characterization is still lacking for systems whose time evolution is influenced by factors which are not under control and appear as random parameters or random noise. We show in this paper how to find the correct indicators for complexity in the information theory context. The crucial point is that the answer is twofold depending on the fact that the random parameters are measurable or not. The content of this apparently trivial observation has been often ignored in literature leading to paradoxical results. Predictability is obviously larger when the random parameters are measurable, nevertheless, in the contrary case, predictability improves when the unknown random parameters are time correlated.
\end{abstract}

In a number of systems the dynamics is influenced by uncontrolled parameters which are intrinsically random or cannot be predicted with necessary precision. The evolution of a system of this type is described in the framework of random dynamical systems, word which indicates in the present paper also dynamical systems with noise.

A dynamical system can be eventually studied by means of the associated symbolic dynamics, which, in this case, correspond to a stochastic process with random conditional probabilities, i.e. probabilities which depend on the same stochastic parameters.

The obvious thing is that the possibility of forecasting the future evolution strongly depends on the possibility of measuring the parameters. The same model will have a different complexity (predictability) according to the fact that the measure is feasible or not. Even if the content this observation appears trivial it is often ignored in literature. For example, most frequently it is used a definition of complexity which considers the separation of nearby trajectories [1 3 , under the same realization of the noise. This definition implicitly assumes that the realization is known and should not be used when the contrary happens, has often it is. For example, the phenomenon of noise induced order [3] should be not considered a reduction of complexity when the random disturbance is non measurable.

A better characterization of complexity for dynamical systems with unmeasurable randomness has been recently found out for many physically relevant cases 任 7 .

In this paper we show how to find proper indicators of complexity in the two cases of measurable (accessible information) and non measurable (inaccessible information) randomness. We also show, with an example, that in case the stochastic parameters have memory, part of the inaccessible information is encoded in the dynamics of the system and can be recovered. In other words, the gap between the two indicators of complexity reduces when the random parameters are time correlated.

Let us start with some basic definition for the non random case also in order to establish the notation. Let us assume that the state of the system is identified by the vector $y(t)$ which evolves as a deterministic dynamical system according to $y(t+1)=f(y(t))$. The corresponding phase space can be partitioned in regions indexed by a symbol $x$. The associated symbolic dynamics ..., $x(1), x(2), . ., x(t), .$. is the realization of a stochastic process with memory, i.e. the probability that the system is in $x(t+1)$ depends on its past history .., $x(1), x(2), . ., x(t)$.

The best characterization of predictability of a process with memory can been found in the information theory context, and it is the Shannon entropy [8]. Assume that $\rho\left(\mathbf{x}_{n}\right)$ is the probability that the sequence of $n$ symbols $x(t+1), . ., x(t+n)$ equals $\mathbf{x}_{n} \equiv x_{1}, . ., x_{n}$, in this case

$$
H_{n}=-\sum_{\left\{\mathbf{x}_{\mathbf{n}}\right\}} \rho\left(\mathbf{x}_{n}\right) \log \rho\left(\mathbf{x}_{n}\right)
$$

is the entropy of the sequence. Then, the entropy rate $h_{n}=H_{n+1}-H_{n}\left(n \geq 0, H_{0} \equiv 0\right)$ measures the average information contained in $n$ steps of the process. In fact, the probability that the system is in $x_{n+1}$ if it was in $x_{1}, . ., x_{n}$ is $\rho\left(x_{n+1} \mid \mathbf{x}_{n}\right)=\rho\left(\mathbf{x}_{n+1}\right) / \rho\left(\mathbf{x}_{n}\right)$ and one has

$$
h_{n}=\sum_{\left\{\mathbf{x}_{n}\right\}} \rho\left(\mathbf{x}_{n}\right) e_{n}\left(\mathbf{x}_{n}\right)
$$

where

$$
e_{n}\left(\mathbf{x}_{n}\right)=-\sum_{\left\{x_{n+1}\right\}} \rho\left(x_{n+1} \mid \mathbf{x}_{n}\right) \log \rho\left(x_{n+1} \mid \mathbf{x}_{n}\right) .
$$

Equation (3) measures the information we have on $x_{n+1}$ if we know $x_{1}, . ., x_{n}$ and (2) is the average with respect 
to all possible sequences $x_{1}, . ., x_{n}$. If $h_{n}=0$ (the minimum possible) one has the maximum of information and next step can be predicted with certitude, on the contrary, when $h_{n}$ attains its maximum, no information is available.

Since, more of the past it is known more it is the information, the rate $h_{n}$ decreases when $n$ increases and the Shannon entropy $h=\lim _{n \rightarrow \infty} h_{n}$ measures the information we can extract from all the past history. It should be noticed that for a process which is $l$-step Markovian $h_{n}=h$ for all $n \geq l$. In this case knowledge of more of the last $l$ steps does not help to predict future.

The Shannon entropy of the symbolic sequence associated to a dynamical system is known as Kolmogorov $\epsilon$-entropy [9]. Eventually, by taking the supremum with respect to all possible partitions it is possible to obtain the Kolmogorov-Sinai entropy, which, in turn, equals the sum of positive Lyapunov exponents.

All above definitions deal with probabilities and with the idea that one has to consider many realizations of the process. In practice, what one has to do is simply to consider a single very long realization (much longer than $M^{n}$ ) of the process and to look for the frequency of any of the sequences of length $n$.

The situation is less straightforward when one deals with a random dynamical system

$$
y(t+1)=f(y(t), \omega(t+1))
$$

where $\omega(t+1)$ is a random variable which can be additive noise or a random parameter. In this case, the associated symbolic dynamics have random conditional probabilities. In fact, $x(t+1)$ not only depends on the past history .., $x(1), x(2), . ., x(t)$, but also on the present value $\omega(t+1)$ and the past history ..., $\omega(1), \omega(2), . ., \omega(t)$ of a second stochastic process which account for the uncontrolled random factors. In this case we call the first subordinated process and the second fundamental process. The reason is that for the problem we have in mind, the fundamental process is autonomous, which means that it has memory but $\omega(t+1)$ only depends on its own past history .., $\omega(1), \omega(2), . ., \omega(t)$. Nevertheless, all considerations which follows also holds for the most general case in which the probability for $\omega(t+1)$ also depends on the pasts history of the subordinated process on ..., $x(1), x(2), . ., x(t-1)$. In this more general case the distinction between fundamental and subordinated process is lost from a mathematical point of view, although it will remain very significant from the information point of view as we will see.

The problem is again to quantify the predictability of the subordinated process, but we are now ready to understand that two different kind of situation may arise. In the first the realization of the fundamental process is known (measurable or accessible information), in the second case it is unknown (non measurable or non accessible information). Correspondingly, a different degree of predictability on the subordinated process is expected. More precisely, there is more information and predictability in the first case (smaller entropy rate) and less in the second (larger entropy rate).

Before entering into the problem let us consider a much simpler atemporal analogous which allows for clarifying the information context. Consider a (subordinated) random variable $x$ whose probability $\rho_{\omega}(x)$ depends on the actual value of a second (fundamental) random variable $\omega$. One can think at this problem as if $\rho_{\omega}(x)$ is the conditional probability for $x$ given $\omega$.

The point we would like to focus can be better understood by the following example. Let us consider a coin toss in which the output is $x$, but two different coins may be used. The two coins are individuated by an index $\omega$ and they have different probabilities for $x$. Given this simple situation, two different games may be played; in both of them one has to guess the output of the toss but the rule is different. In the first game the player chooses at random the coin, gives a look at it, makes the guess and tosses; in the second he makes the guess and only after he chooses the coin at random and tosses.

In the first game, at the moment of the guess, $\omega$ is known and the information is measured by the entropy $-\sum_{\{x\}} \rho_{\omega}(x) \log \rho_{\omega}(x)$. Furthermore, since the coin has been chosen at random, the entropy of the game before the start is the average

$$
\tilde{H}=-\sum_{\{x\}}<\rho_{\omega}(x) \log \rho_{\omega}(x)>
$$

One can easily check that $\tilde{H}=H_{x, \omega}-H_{\omega}$, where $H_{x, \omega}$ is the entropy of the couple of random variables and $H_{\omega}$ is the entropy of $\omega$.

If the second game, $\omega$ is unknown at the moment of the guess, and the utilized probability is the average $<\rho_{\omega}(x)>$ Therefore, the information content is measured by

$$
H=-\sum_{\{x\}}<\rho_{\omega}(x)>\log <\rho_{\omega}(x)>
$$

The inequalities $\tilde{H} \leq H \leq H_{x, \omega}$ hold. The first inequality trivially means that in the second game one has less information than in the first. The equality holds only in the case of independence between the two random variables. In turn, the second inequality becomes an equality only in case of complete dependence of the two variables i.e. at given value $x$ corresponds deterministically only a single value $\omega$.

The above discussion, although very simple, allows for the treatment of the intriguing case of stochastic process with random probability and, therefore, also the case of dynamical systems with noise.

In the first scenario, once probabilistically generated, the sequence $. ., \omega(1), . ., \omega(t), .$. can be measured and it 
can be treated as a known ordinary time dependent function. In consequence, the average entropy of a sequence of length $n$ is given by

$$
\tilde{H}_{n}=-\sum_{\left\{x_{1}, \ldots, x_{n}\right\}}<\rho_{\omega}\left(\mathbf{x}_{n}\right) \log \rho_{\omega}\left(\mathbf{x}_{n}\right)>
$$

since $\rho_{\omega}\left(\mathbf{x}_{n}\right)$ is the probability for the sequence $x_{1}, . ., x_{n}$ given the realization of the fundamental process.

The entropy rate $\tilde{h}_{n}=\tilde{H}_{n+1}-\tilde{H}_{n}$ represents now the average information one has on $x(t+1)$ if one knows its previous $n$ steps $x(1), . ., x(t)$ and also one knows all the fundamental process from the most recent $\omega(t+1)$ to the more remote past.

In this case, in analogy with the atemporal example, one can easily show that the Shannon entropy $\tilde{h}=$ $\lim _{n \rightarrow \infty} \tilde{h}_{n}$ is the difference between the Shannon entropy associated with the couple process and that the Shannon entropy of the fundamental process i.e. $\tilde{h}=h_{x, \omega}-h_{\omega}$. Therefore, from a practical point of view, what one has to do is to generate or to consider a very long sequence of both the processes and then measure the frequency of the sequences $\left(x_{1} ; \omega_{1}\right), . .,\left(x_{n} ; \omega_{n}\right), .$. and, separately, of the sequence $\omega_{1}, . ., \omega_{n}, .$. .

Furthermore, $\tilde{h}$ is nothing else that the characterization of complexity which refers to the separation of two nearby trajectories corresponding to the same realization of the fundamental process (noise). Notice, in fact, that one also trivially has $h=\lim _{n \rightarrow \infty} \tilde{H}_{n} / n$. Since $\tilde{H}_{n} / n$ is an average of a quantity which is non random in the limit one can replace the average by simply considering the typical value, i.e. that value corresponding to a single realization of the fundamental process. This realization only plays the role of an ordinary function of time. The Pesin relation assures, in this case, that $\tilde{h}$ equals the sum of positive Lyapunov exponents corresponding to the separation of trajectories under the same realization of the fundamental process. This quantity is often taken as a measure of complexity for chaotic noisy systems. Nevertheless, let us stress once again that it is a measure of predictability only if the noise realization itself can be measured with infinite precision.

Let us consider the more realistic case in which on the contrary $\omega(t)$ cannot be measured. In a way which is completely analogous to (6) one finds that the entropy associated to a sequence of length $n$ is

$$
H_{n}=-\sum_{\left\{x_{1}, . ., x_{n}\right\}}<\rho_{\omega}(x)>\log <\rho_{\omega}(x)>
$$

and the entropy rate $h_{n}=H_{n+1}-H_{n}$ describes now the information content of $n$ steps of the subordinated process when the fundamental process is unknown. The Shannon entropy $h=\lim _{n \rightarrow \infty} h_{n}$ is the maximum information which is available from the past.

In practice, in order to obtain $h_{n}$, one has to generate the long sequence .., $x(1), x(2), . ., x(t), .$. and measure the frequency of the sequences without regarding at the fundamental process.

We stress that this characterization of predictability may differ a lot from the first one. For example, even non chaotic dynamical systems with negative or vanishing Lyapunov exponents $(\tilde{h}=0)$ may have a positive Shannon entropy $h$ and to be largely unpredictable. More generally the inequality $\tilde{h} \leq h \leq h_{x, \omega}$ holds. The qualitative understanding of this inequality is straightforward since $\tilde{h}$ refers to the case in which the additional information on the fundamental process is available. On the other side, it is easy to check that $h$ is smaller or equal than the entropy of the couple process. Since $\tilde{h}=h_{x, \omega}-h_{x}$ one can also rewrite $h_{x, \omega}-h_{x} \leq h \leq h_{x, \omega}$. The first inequality becomes an equality only when the two process are reciprocally independent, while the the second one when they are deterministically linked.

Unfortunately, while one has that $\tilde{h}$ can be computed by means of the Lyapunov exponents of a typical trajectory, there is not a corresponding general recipe for $h$. Nevertheless, a very sensitive approximate approach based on the use of the rate of separation of two nearby trajectories which correspond to two different realization of the fundamental has been recently proposed [4] .

Let us now give a simple example which may help to understand an important point: a subordinated process which is memoryless when the realization of the fundamental process is given, may have a long memory when, on the contrary, the fundamental process is unknown. This is because part of the information carried by the fundamental is encoded in the history of the subordinated. The long memory of the subordinated may be induced even if the fundamental itself is Markov. The more the fundamental is correlated, longer is the memory of the subordinated. As a consequence, the difference between $h$ and $\tilde{h}$ decreases when the correlation of the fundamental increases and it disappears in case this correlation is complete.

Let us consider, as an example, the following simple dynamical system

$$
y(t+1)=2^{\omega(t+1)} y(t) \quad \bmod .1
$$

where the $\omega(t)$ are Markovian variables which can take the two possible values 0,1 with equal probability and which persist in their value with probability $p$.

Now let us consider the most trivial partition of the accessible phase space: the two intervals $[0,1 / 2),[1 / 2,1)$ identified respectively by the symbols $x=0$ and $x=1$. The symbolic dynamics is very easy to construct: when $\omega(t+1)=0$ then $x(t+1)=x(t)$, when $\omega(t+1)=1$ then $x(t+1)=0,1$ with same probability $1 / 2$.

Than it is straightforward to obtain, independently on $p$ the entropy rate $\tilde{h}_{n}=\frac{1}{2} \log 2$ for $n \geq 1$. This entropy equals the typical rate of separation of the trajectories.

On the contrary $h_{n}$ strongly depends on $p$. If $p=0$ than $h_{n}=-\frac{3}{4} \log \frac{3}{4}-\frac{1}{4} \log \frac{1}{4}$ for $n \geq 1$. This result can be 
easily interpreted in this way: the averaged probability that $x(t)$ persist in its value is $3 / 4$, while the averaged probability for a change is $1 / 4$.

In fig $1 . h_{n}$ is plotted for three different values of $p \neq 0$ and compared both $\tilde{h}_{n}$ and the value of $h_{n}$ corresponding to $p=0$. When $p \neq 0$, one see from fig 1 . that $h_{n}$ is strictly monotone and converges to $h$ in more than a single step. This behavior implies that in absence of informations on the fundamental the subordinated is not Markovian even if the couple process itself is Markovian. This behavior also implies that when $n$ increases, the knowledge of the subordinated trajectory can be used to better forecast.

Furthermore, one observes in fig. 1 that the asymptotic difference $h-\tilde{h}$ reduces when $p$ increases and almost vanishes for very large $p$. This is of easy understanding since, in practice, one guesses the value of $x(t+1)$ by looking at the length of the previous sequence of symbols with same persistent value. The larger is $p$, the longer are these sequences which carry information.

In other words, since the more it is possible to use of the past, the more the information is recovered, the difference $h-\tilde{h}$ reduces when $p$ increases.

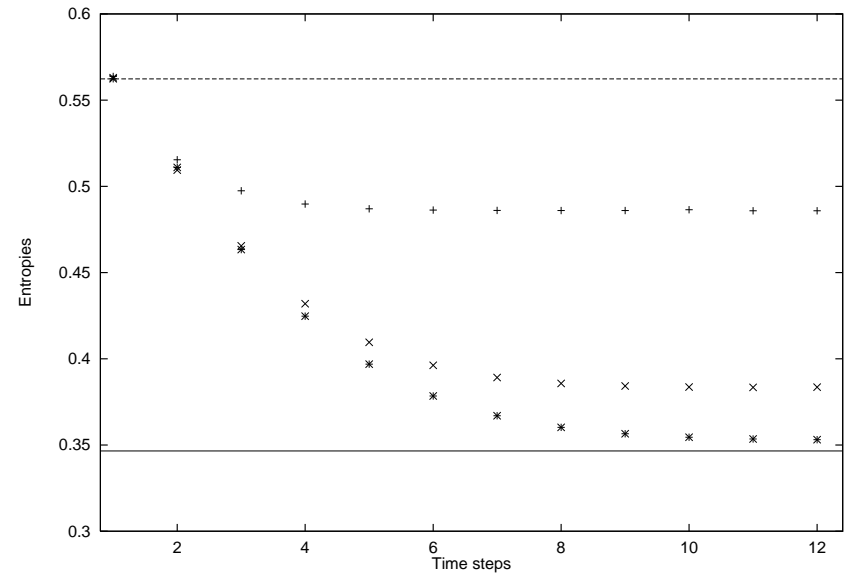

FIG. 1. The entropy rate $h_{n}$ is plotted for three different values of $p: \quad p=.9$ (crosses), $p=.99$ (slanting crosses), $p=.999$ (asterisks). For comparison, both $\tilde{h}_{n}$ (line) and the value of $h_{n}$ corresponding to $p=0$ (dots) are reported.

This example shows that the topic of this paper may be quite relevant when one deal with historical data, i.e. single non reproducible sequences of symbols, where the joint probabilities depend on some stochastic parameters. For example this phenomenology is typical of finance where the random parameters reflect economic factors which may be unknown to a given investor. Investors which trust in fundamental analysis strongly believe that all information is reflected in the price dynamics. The previous example also shows the limit of this point of view. In fact, the missing information is only partially reflected by data and only information on longly persis- tent macro-economic factors can be totally recovered.

Let us resume the result of this paper as follows: if the fundamental process is known, the predictability is measured by $\tilde{h}$ which can be computed in practice as the Shannon entropy of a single long realization of the couple process minus the Shannon entropy of a single long realization of the fundamental process. This entropy equals the sum of positive Lyapunov exponents associated to the separation of nearby trajectories under the same realization of the noise;

if the fundamental process is unknown, the predictability is measured by $h$ which can be computed in practice as the Shannon entropy of a single long realization of the subordinated process. Unfortunately, there is not an exact recipe which allows for the calculation of $h$ by means of Lyapunov exponents and some more refined approximate techniques have to be used [4].

The author is indebted with Angelo Vulpiani for an infinite number of discussion on complexity, chaos and predictability. He also thanks Roberto Baviera, Michele Pasquini and Yi-Cheng Zhang for discussion and suggestions concerning the topic of this work. This research has been partially supported by the European Network contract FMRXCT980183.

[1] J.P. Crutchfield, D.J. Farmer and B.A. Huberman, Phys. Rep 92, (1982), 45.

[2] A.R. Bulsara, E.W. Jacobs and W.C. Schieve, Phys. Rev. A 42, (1990), 5837

[3] K. Matsumoto and I. Tsuda, J. Stat. Phys. 31, (1983), 87

[4] G. Paladin, M. Serva and A. Vulpiani, Phys. Rev. Lett. 74, (1995), 66.

[5] V. Loreto, G. Paladin and A. Vulpiani, Phys. Rev. E 53, (1996), 2087.

[6] V. Loreto, M. Serva and A. Vulpiani, Proceedings of Trends in complexity, (Yokohama 1996). Int. J. Mod. Phys. B 12, (1998), 225.

[7] V. Loreto, G. Paladin, M. Pasquini and A. Vulpiani, Physica A 232, (1996), 189.

[8] C.E. Shannon and W. Weaver, The mathematical theory of communications, Univ. of Illinois Press, Urbana (1949).

[9] A.N. Kolmogorov, IRE Trans. Inf. Theory 1, (1956), 102. 